\title{
Comment préférez-vous votre guide ou mandrin pour intuber ? Long ou court, plein ou creux, dur ou mou, droit ou béquillé ?
}

\author{
How do you prefer your intubation bougie? Long or short, full or hollow, hard \\ or soft, straight or bent?
}

\section{Patrick Schoettker $^{a, *}$, Sina Grape ${ }^{a, b}$}

\author{
a Service d'anesthésie, centre hospitalier universitaire Vaudois (CHUV), rue du Bugnon, \\ CH 1011 Lausanne, Suisse \\ b Service d'anesthésie et de réanimation, hôpital de Sion, CH 1950 Sion, Suisse
}

\section{MOTS CLÉS \\ Intubation ; \\ Bougie ; \\ Mandrin ; \\ Intubation difficile ; \\ Aides à l'intubation ; \\ Guide long}

\begin{abstract}
Résumé
But. - Les guides et mandrins sont des dispositifs régulièrement utilisés pour la gestion des voies aériennes difficiles dans différents scénarios. Leur utilisation en première intention est proposée par un grand nombre d'algorithmes. Leur forme et techniques d'utilisation évoluent régulièrement. Notre but est de fournir au clinicien une mise à jour concernant les caractéristiques, indications, techniques d'utilisation et les dangers des différents guides et mandrins utilisés comme aides à l'intubation.

Méthode. - Nous avons effectué une recherche systématique de la littérature jusqu'en mai 2015, sans restrictions de langue. Les deux auteurs ont retiré, de façon indépendante, toutes les études, rapports de cas et séries avec les mots clés "bougie », " gum elastic bougie », «stylet », « airway management », «tracheal tube introducer », « intubation aid », « extubation catheter », « bougie élastique », « mandrin », « stylet d'intubation », « management des voies aériennes », « introducteur », « aide à l'intubation » et « mandrin d'extubation ».

Résultats. - Nous avons trouvé principalement des études observationnelles et rétrospectives avec au total un petit nombre de sujets. Ces données ont été synthétisées dans une revue narrative.

Conclusion. - À ce jour, de nombreux guides et mandrins existent pour assister à la gestion de la voie aérienne difficile. Chacun d'entre eux possède des indications différentes, avec une
\end{abstract}

\footnotetext{
* Auteur correspondant.

Adresse e-mail : patrick.schoettker@chuv.ch (P. Schoettker).
} 


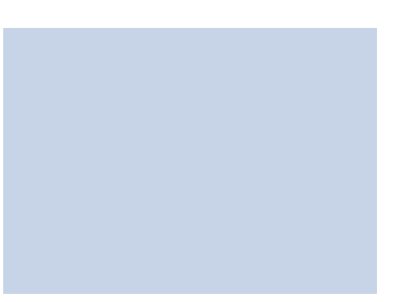

spécificité variable. Les médecins anesthésistes, réanimateurs et urgentistes se doivent d'avoir une connaissance et compréhension maximale de ces différents dispositifs afin d'utiliser le bon instrument au bon moment et de manière adaptée à la situation. Ainsi une prise en charge optimale de la voie aérienne difficile pourra être atteinte.

(c) 2016 Elsevier Masson SAS. Tous droits réservés.

\section{KEYWORDS \\ Intubation; \\ Bougies; \\ Stylets; \\ Difficult intubation; \\ Intubation aids}

\section{Introduction}

La prise en charge de patients avec des voies aériennes difficiles peut se compliquer de lésions sévères [1,2] et mettre rapidement en jeu le pronostic vital du patient [2,3]. Quand l'orifice trachéal ne peut être visualisé d'une manière adéquate par laryngoscopie directe, deux techniques simples et efficaces sont à notre disposition : 1 - préformer à l'aide d'un mandrin malléable court (stylet) le tube endotrachéal (TET) et le diriger antérieurement [4] ; 2 - insérer à l'aveugle un guide long béquillé et idéalement à extrémité souple (introducteur à extrémité flexible), puis glisser un TET sur cet introducteur selon la technique de Seldinger [5].

Les stylets et améliorent le taux de succès de l'intubation dans des situations de voies aériennes difficiles ; ainsi leur utilisation précoce est mise en avant dans la majorité des recommandations de prise en charge [6-8]. Même s'ils n'ont pas été destinés à fonctionner comme échangeur de tube [9-11] ou mandrins d'extubation [12], certains guides et introducteurs sont utilisé à cet effet et ont par la suite été modifiés spécifiquement afin de permettre de procéder à ces manœuvres d'une manière aussi sûre que possible. Finalement, le place que prend la technique de la vidéolaryngoscopie dans la gestion de l'intubation a ouvert de nouvelles pistes pour l'utilisation des mandrins courts et des guides longs béquillés [13-15].

De nos jours, de nombreuses modifications des dispositifs originaux sont proposées. De nouveaux types de mandrins, guides longs béquillés, d'introducteurs ou guides spécifiques pour l'extubation, ont fait leur apparition sans que leur rôle et leurs avantages soient validés scientifiquement dans la gestion de la voie aérienne difficile.

Le but de cette revue est de fournir une mise à jour concernant les caractéristiques, indications, techniques d'utilisation et dangers des différents mandrins et guides longs.

\section{Méthode}

Les deux auteurs ont conduit, de façon indépendante, une recherche systématique de la littérature (MEDLINE, EMBASE). Toutes les revues et études cliniques randomisées contrôlées, publiées de 2005 jusqu'en mai 2015, et sans restriction de langue ont été recherchées. Les mots clés pour la recherche étaient «bougie », « gum elastic bougie », « stylet », « airway management », « tracheal tube introducer », « intubation aid », « extubation catheter », «bougie élastique », « mandrin », « stylet d'intubation », 
« management des voies aériennes », « introducteur », « aide à l'intubation » et «mandrin d'extubation ». En plus des revues et études randomisées contrôlées identifiées, les rapports et séries de cas ont été inclus si nécessaire, en particulier pour la partie concernant les complications en rapport avec les guides longs. Nous avons identifié 396 articles, dont 17 duplicatas ont été exclus ; 323 articles ont été rejetés après avoir lecture du titre ou de l'abstract. Une évaluation détaillée de 56 articles a été effectuée, dont 19 ont été rejetés car non contributifs. Les 37 articles restants ont été évalués et leurs données sont résumées dans une revue narrative.

\section{Guides ou mandrins pour la gestion des voies aériennes}

\section{Guides longs pour intubation}

Les caractéristiques qui décideront du choix d'un guide long spécifique dans une situation clinique incluent son diamètre, sa longueur, sa structure, sa flexibilité, le fait d'être destiné à l'usage unique ou réutilisable, sa forme, le type d'extrémité distale (angulé/coudé ou droit), sa pointe flexible ou dure et le fait de comporter un lumen creux ou non. La Fig. 1 illustre un choix de guides longs couramment utilisés.

\section{Les guides longs type Eschmann}

On attribue à Sir Robert Reynolds Macintosh en 1949 l'invention du premier guide long pour intubation. Son prototype était à l'origine un cathéter de dilatation urétrale que Macintosh modifia légèrement. Ainsi, il le nomma «Gum elastic bougie (GEB) - bougie élastique en gomme ", nom encore utilisé à ce jour, même si les guides longs contemporains ne sont pas forcement élastiques, ni constitué de gomme, ni utilisés selon la technique de la bougie. Basé sur la description de Macintosh, PH Venn dessina dans les années 1970 le premier guide long spécifiquement destiné à assister l'intubation endotrachéale
[16]. Ce guide long fut ensuite produit par la compagnie anglaise Eschmann et commercialisé sous divers noms, tels que « introducteur d'Eschmann » ou « guide long selon Eschmann » ou « long mandrin d'Eschmann ». Aujourd'hui, les guides longs de type Eschmann mesurent $600 \mathrm{~mm}$ et ont un diamètre externe de $5 \mathrm{~mm}$, ce qui permet une insertion dans un tube endotrachéal (TET) de taille 6 ou plus. L'extrémité distale est typiquement angulée (béquillée) à $40^{\circ}$. Une texture en polyester leur procure une certaine raideur tout en offrant de l'élasticité et facilite en même temps leur insertion et manipulation. Lorsqu'ils sont pliés, les guides longs de type Eschmann retiennent ainsi leur forme (effet mémoire [17]).

Les guides longs de type Eschmann sont ainsi introduits à travers les cordes vocales sous vue directement ou partiellement ou même à l'aveugle. La pointe béquillée dirigée vers le haut facilite le passage dans la trachée et donnait une sensation tactile (clics) quand le guide glissait sur les anneaux trachéaux pour se terminer par le signe de l'arrêt (hold-up) lorsqu'il s'impactait dans les voie aériennes de plus petit calibre [5]. Une fois en place, il est possible de glisser un TET et d'enlever l'introducteur secondairement. Cette manière de rechercher activement les contacts avec la muqueuse, voire les voies aériennes de petits calibres, n'est actuellement plus recommandée, notamment en raison de lésions potentiellement induites par ces manœuvres.

Plusieurs séries de cas décrivent également le rôle facilitateur des guides longs pour l'insertion de dispositifs supra-glottiques $[18,19]$. Alors que le guide original était destiné à utilisation multiple en étant re-stérilisé, les guides modernes sont pour la plupart à usage unique, tels que les guides Portex (Smiths medical Intl Ltd., Kent, Royaume-Uni), ou guides VBM (VBM Medizintechnik GmbH, Sulz a. N, Allemagne).

Le «traffic-light bougie » ou guide long type «tricolore » est un prototype récent qui utilise un code couleur signalant sa profondeur d'insertion. Il est destiné à prévenir un traumatisme des voies aériennes lié à une insertion trop profonde [20]. La zone verte indique une profondeur d'insertion sûre $(<21 \mathrm{~cm})$, jaune indique un contact possible avec la carène et la couleur rouge signale un risque élevé

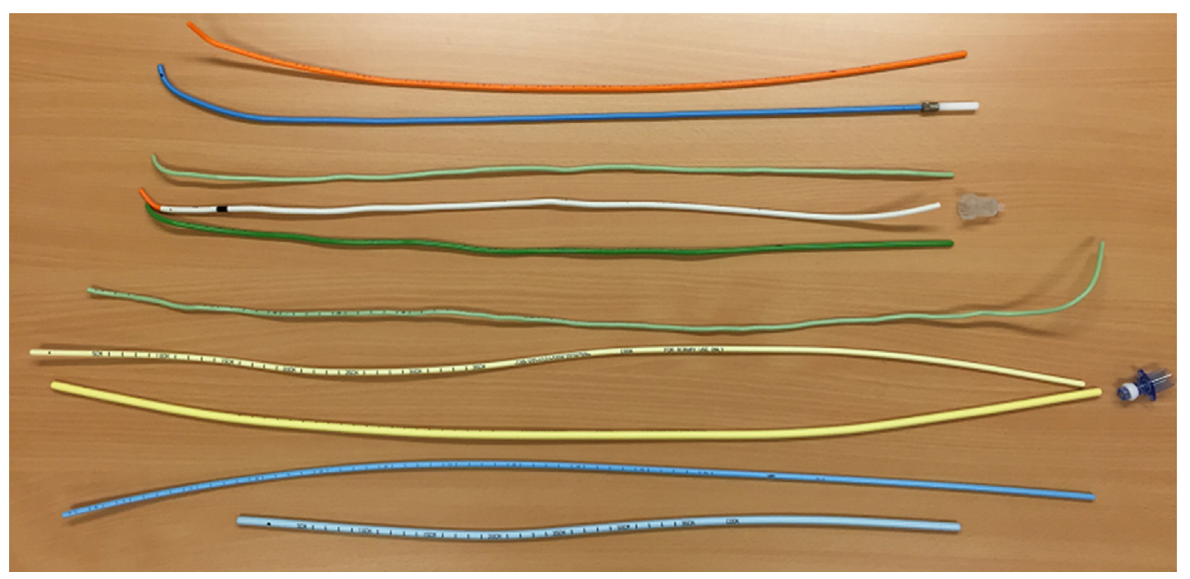

Figure 1. Choix de guides longs. Légende (de haut en bas) : guide long type Eschmann, VBM ; guide long pour intubation selon Frova, Cook Medical ; Muallem endotracheal tube stylet (METTS), VBM; S-guide with universal connector, VBM ; Muallem endotracheal tube introducer (METTI), VBM ; Rivier airway introducer, VBM ; Cook Airway exchange introducer $14 \mathrm{Fr}$, Cook Medical ; Cook airway exchange introducer $8 F r$ with rapi-fit adaptor, Cook Medical ; tube exchanger, VBM ; Aintree intubation catheter, Cook Medical. 
d'impact dans les voies aériennes distales. L'adéquation des ces distances à néanmoins été questionnée [21].

\section{Les guides longs à extrémité angulée avec lumen interne pour oxygénation}

Un sondage parmi des anesthésistes et urgentistes anglais en 2009 a montré que le guide long type «Frova » (Cook Critical Care, Bloomington, Indiana, EEUU) était leur guide à usage unique, préféré [22]. Sa longueur de $650 \mathrm{~mm}$ avoisine celle des guides type Eschmann, mais sa pointe est angulée à $65^{\circ}$ au lieu de $40^{\circ}$, permettant un meilleur coulissage sous l'épiglotte et ainsi une introduction plus aisée dans la trachée. Des modèles pédiatriques sont également disponibles dans des longueurs et tailles différentes, permettant le maniement avec des TET dès la taille 3 .

Construit en polyéthylène, le guide «Frova » possède un certain effet mémoire. Il a été conçu avec une rigidité suffisante pour transformer une force directe appliquée à son extrémité proximale en un mouvement antérieur et vers le haut de sa pointe, résultant en un déplacement antérosupérieur, en direction de l'ouverture trachéale, en cas de contact avec les cartilages aryténoïdes [23].

En plus de faciliter l'intubation, le Frova permet l'oxygénation en cours de procédure. L'extrémité proximale peut être connectée à une source d'oxygène à l'aide d'un adaptateur spécifique, l'oxygène pouvant ainsi être insufflé à travers la lumière intérieure du guide jusqu'à l'extrémité distale et ses ouvertures latérales. L'oxygénation ou la ventilation type « jet » est également possible. Une évaluation de l'équipement disponible pour la gestion des voies aériennes dans les hôpitaux anglais en 2009 a relevé que les guides longs types Eschmann ou Frova étaient les seuls disponibles dans 395 des institutions, alors que de nombreux autres types de guides ou stylets étaient à disposition, un certain nombre étant même qualifié d'inadéquat [22].

\section{Autres type de guides longs d'intubation}

Différents guides longs existent dont la composante centrale (noyau) en polyester a été remplacée par du métal afin de renforcer l'effet mémoire. Le METTS [24] (Muallem ET Tube Stylet, VBM Medizintechnik GmbH, Sulz a. N., Allemagne) combine un noyau métallique relativement dur avec une pointe préformée et flexible. Il existe en taille adulte et pédiatrique, permettant l'utilisation de TET dès la taille 3,5. Le METTI [25] (Muallem ET Tube Introducer, VBM Medizintechnik GmbH, Sulz a. N., Allemagne) avec $800 \mathrm{~mm}$ est plus long et peut servir à la fois comme guide pour l'intubation difficile ou comme échangeur de tube. Aucun des deux ne possède néanmoins une lumière permettant une oxygénation pendant la procédure. Ces guides longs pour l'intubation sont à usage unique.

\section{Les guides échangeurs de tubes}

Ces guides longs ont été spécifiquement dessinés pour être introduits à travers un TET, un masque laryngé ou enfilés sur une fibre optique et permettre un accès à la voie aérienne, même si le TET original a été enlevé. Afin d'être utilisés comme échangeurs de tubes, ils se doivent d'être suffisamment long (plus de 2 fois la longueur du tube) afin de garder une sécurité lors de la procédure d'emploi. Une approche «pas à pas », rigoureuse et codifiée permet un échange de tube tout en optimalisant les conditions de succès et de sécurité. Une oxygénation optimale, suivie d'une insertion du guide échangeur dans le tube existant sous contrôle visuel, un retrait du tube tout en maintenant le guide en place suivi d'une re-intubation sur guide en place sous contrôle visuel par laryngoscopie ou vidéolaryngoscopie permet un déroulement optimal de cette procédure [26].

\section{Guides mixtes, échangeurs longs et droits avec lumière d'oxygénation et intubateurs}

Le guide d'échange Cook [27] (Cook Critical Care, Bloomington, Indiana, États-Unis) est à usage unique, d'une longueur de $800 \mathrm{~mm}$, avec un connecteur à oxygène proximal et des ouvertures distales latérales qui permet une oxygénation pendant la procédure d'échange. Son extrémité distale est modérément souple pour un passage facilité à travers un TET. Une graduation centimétrique aide à guider la profondeur d'insertion, et plusieurs tailles sont disponibles, permettant une utilisation pour tubes endotrachéaux de taille 3 et supérieures [28]. L'échangeur de TET VBM est une alternative à partir de tubes de taille 4 ou plus (VBM Medizintechnik GmbH, Sulz a. N, Allemagne, http://www.vbm-medical.com/products/airwaymanagement/stylets-introducers-tube-exchangers/).

\section{Guides échangeurs longs et droits avec lumen pour une fibre optique}

Le guide d'intubation Aintree [29] (Cook Critical Care, Bloomington, Indiana, États-Unis) est un dispositif à usage unique, avec une connexion proximale permettant l'adaptation d'une source d'oxygène, associé à une extrémité distale non tranchante et fenêtrée, permettant un débit de gaz suffisant. Le diamètre interne de $4,7 \mathrm{~mm}$, relativement large, et une longueur de 56 centimètres, permettent l'insertion d'une fibre optique tout en conservant la mobilité distale du fibroscope. Le guide d'Aintree est positionné sous contrôle fibroscopique et un TET de taille 7 ou plus peut être inséré tout en le gardant comme guide. Ainsi, en plus de permettre une intubation primaire, l'Aintree peut être utilisé pour l'échange de TET tout en étant positionné par fibroscopie. Comme il s'adapte au travers d'un dispositif supra-glottique, il peut également servir de guide à l'intubation trachéale tout en administrant de l'oxygène au patient [30-32]. Son développement a été intimement lié à la volonté de permettre une intubation assisté par la fibre optique, tout en gardant à tout moment un contrôle de la voie aérienne. Ainsi, la présence d'un dispositif extraglottique ne gêne en rien l'insertion de ce guide monté sur un fibroscope, permettant une visualisation de la glotte, des anneaux trachéaux et de la carène. Par la suite, le fibroscope et le dispositif extra-glottique sont retirés, laissant l'Aintree dans la trachée. À l'aide d'un laryngoscope, une TET est finalement inséré sur le guide, qui sera retiré à la fin de l'intubation. Ce guide échangeur, long et droit, permet ainsi une utilisation comme aide à l'intubation et comme échangeur de tube. 


\section{Guides pour l'extubation}

L'intérêt autour de la prise en charge des voies aériennes s'est concentré sur l'intubation et l'accès trachéal. Plus récemment, l'existence de complications menaçant la vie du patient, en relation avec des difficultés dues à l'extubation, ont mené à une réflexion globale autour de la prise en charge des voies aériennes. Les premières recommandations de gestion de l'extubation [33] ont souligné l'importance de la planification de ce geste, de l'adéquation du matériel à disposition et de stratégies adaptées en fonction de la situation clinique. Dans un audit récent, jusqu'à $30 \%$ des problèmes respiratoires étaient liés à l'extubation, particulièrement chez les patients intubés sur des périodes prolongées [3].

Afin de gérer des situations à haut risques pour la phase d'extubation, l'utilisation de guides longs et creux, de petits diamètres, initialement destinés à l'échange de petits tubes, a permis une amélioration de la sécurité dans quelques cas [34-36].

La mise à disposition sur le marché d'un set spécifique, destiné principalement à la prise en charge de l'extubation difficile (Cook Critical Care, Bloomington, Indiana, ÉtatsUnis) va potentiellement améliorer la gestion de cette situation à haut risque. Ce set contient un mandrin fin en nitinol flexible et d'une longueur de $1450 \mathrm{~mm}$, qui ne se plie pas et permet ainsi un passage aisé d'un cathéter de réintubation creux de $830 \mathrm{~mm}$ sur ce fil. En cas de nécessité, le patient pourra être re-intubé, en utilisant le cathéter de ré-intubation comme guide.

Quand la situation est jugée à haut risque et avant l'extubation, le mandrin fin en nitinol est positionné à l'intérieur du tube trachéal. Le patient est alors extubé et une évaluation de la situation clinique permet une stratégie dédiée. Au vu de la finesse du mandrin en nitinol, celuici est généralement mieux toléré que tout autre dispositif trachéal. Si la nécessité d'une re-intubation venait à se préciser, le cathéter de ré-intubation est alors inséré sur ce mandrin, pour finalement procéder à une nouvelle intubation [37].

\section{Les mandrins}

Les mandrins d'intubation (aussi appelés stylets) sont insérés dans un TET avant l'intubation. Idéalement, l'extrémité distale du mandrin est positionnée juste avant l'œil de Murphy, tandis que le bout proximal doit être ancré sur le connecteur du TET. Le stylet avec le TET peut ensuite être préformé en fonction de l'anatomie et de la situation clinique. L'angulation distale fréquemment reportée comme idéale s'approche des $35^{\circ}$, en maintenant le reste du stylet bien droit. Cette technique «stylet en forme de canne de hockey » [38] laisse l'extrémité distale du TET libre pour des manipulations ou des mouvements spécifiques. Ainsi, en synchronisant adroitement le stylet, une rétraction de ce dernier et une rotation du tube est possible. La « danse du tube » représente une série de mouvements induits par cette gestuelle [39]. Lors de l'intubation, le stylet doit idéalement être retiré dès que le tube endotracheal passe les cordes vocales afin de diminuer au maximum les lésions possibles. En aucun cas, un tel stylet ne doit dépasser du TET, son extrémité distale étant par défaut une structure rigide avec

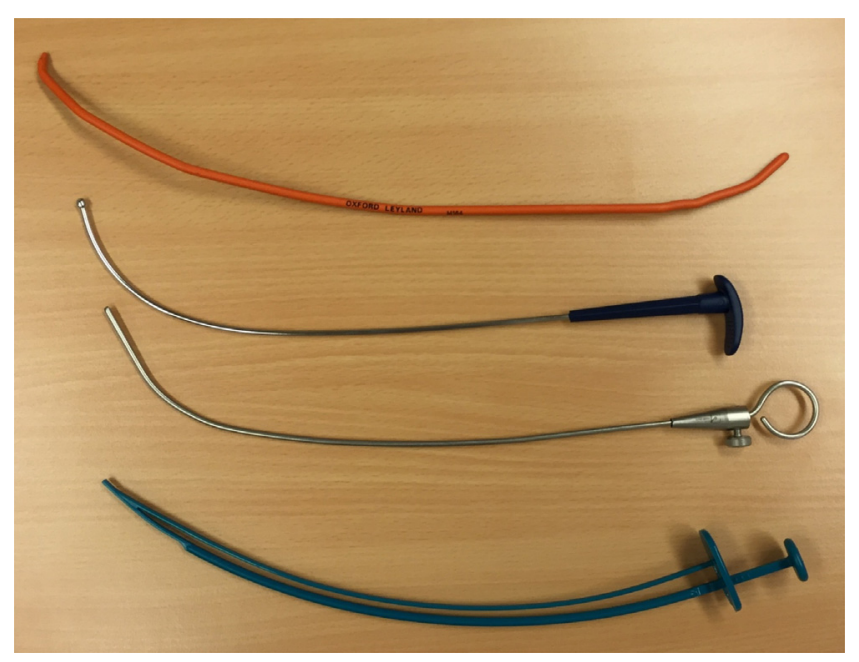

Figure 2. Exemples de mandrins pour intubation. Légende (de haut en bas) : Oxford Leyland stylet ; Gliderite stylet, Verathon; stylet métallique, centre hospitalier universitaire Vaudois, Lausanne, Suisse ; Parker articulating stylet.

haut potentiel de morbidité. La Fig. 2 illustre un choix de mandrins pour intubation.

\section{Les mandrins métalliques}

Les mandrins standards sont des tiges métalliques courtes, possédant une certaine malléabilité en relation avec le type et l'épaisseur de métal utilisé. Différents modèles existent, se caractérisant notamment par une surface faite de matériaux divers pour un coulissage facile (p.ex. mandrins Portex, Smiths medical International Ltd., Kent, Royaume-Uni). Ils sont disponibles dans des longueurs et diamètres variables, destinés à un usage unique ou multiple. Dans de nombreuses institutions, l'usage de tels stylets est recommandé pour les intubations à séquence rapide ou en milieu pré-hospitalier $[40,41]$.

La technique consistant à préformer un TET à l'aide d'un stylet et d'effectuer l'intubation par vidéolaryngoscopie, s'est fortement répandue. Des stylets tels que le Gliderite ${ }^{\odot}$, expressément destiné à assister l'intubation trachéale avec le Glidescope ${ }^{\odot}$ [42], sont ainsi mis à disposition par les producteurs de vidéolaryngoscopes utilisé dans la gestion de la voie aérienne.

Les mandrins traditionnels, existant avant l'arrivée sur le marché d'appareils intégrant des techniques de vidéolaryngoscopies, semblent avoir un taux de succès comparable à celui des stylets spécifiques. Néanmoins, de nombreux cas de complications dues notamment au Gliderite par exemple, sont relatés dans la littérature lors d'un usage inapproprié.

\section{Les mandrins non métalliques}

Les mandrins articulés Parker (Flex-It ${ }^{T M}$ ) ou stylet directionnel type Schroeder [43] sont des mandrins en plastique à usage unique, insérés directement dans le TET. La courbure distale du tube est ensuite modifiée directement pendant l'intubation, en exerçant une pression spécifique avec le pouce sur un ressaut du mandrin $[44,45]$. Aucune étude n'a démontré la supériorité de ces stylets à ce jour et le développement continu d'outils et de techniques améliorant l'intubation ont conduit à l'abandon de ces stylets. 
Tableau 1 Caractéristiques des guides longs et mandrins.

\begin{tabular}{|c|c|c|}
\hline But & Type & Exemple \\
\hline \multirow[t]{2}{*}{ Intubation } & Mandrins & Gliderite $^{\circledR}$, VBM malleable Stylet ${ }^{\circledR}$ \\
\hline & Guides longs & Eschmann bougie, METTI ${ }^{\circledR}$ \\
\hline \multirow[t]{4}{*}{ Intubation \& oxygenation } & Guides longs béquillés creux pour & Frova introducer ${ }^{\circledR}$ \\
\hline & oxygénation & VBM Introducer ${ }^{\circledR}$ \\
\hline & Guides longs droits pour insertion fibroscope & Aintree catheter ${ }^{\circledR}$ \\
\hline & & VBM Intubation catheter ${ }^{\circledR}$ \\
\hline \multirow[t]{2}{*}{ Échangeur de tube } & Guides longs droits et creux pour & Cook airway exchange catheter ${ }^{\circledR}$ \\
\hline & oxygénation & VBM tube exchanger ${ }^{\circledR}$ \\
\hline Extubation & $\begin{array}{l}\text { Set spécifique avec cathéter en nitinol et } \\
\text { guide long creux dédié }\end{array}$ & Cook staged extubation set ${ }^{\circledR}$ \\
\hline
\end{tabular}

\section{Complications}

Après des décennies d'utilisation dans une variété de situations cliniques de voies aériennes difficiles, il est indiscutable que les guides longs et mandrins peuvent sauver des vies en améliorant le taux de succès de ces intubations malaisées $[46,47]$, tout en maîtrisant les avantages et inconvénients de chacun (Tableau 1). Néanmoins, il faut se rappeler que des séries de cas ont décrit des problèmes sérieux comportant une morbidité considérable, voir mortalité liée à ces dispositifs. Les complications les plus fréquentes sont de nature mécanique, quand une pression excessive est appliquée ou lors d'essais d'intubation multiples. Étant plus rigides, les mandrins, mais aussi les introducteurs à usage unique semblent associés à un taux de complications plus élevé que les introducteurs à usage multiple [48]. Par exemple, un taux de complications jusqu'à $5 \%$ a été rapporté avec le long guide Frova [49]. Les problèmes mécaniques décrits, en relation avec les guides longs incluent les lésions pharyngées [50] ou trachéales [51], la rupture bronchique [52], l'hémorragie alvéolaire [53] et l'hémo-pneumothorax [54]. Les stylets ont également été rendus responsables de perforations palatines ou amygdaliennes [55-57]. La rupture d'un mandrin avec la migration endotrachéale ou pulmonaire des composantes incriminées a également été décrite [58-60]. De même, les guides longs échangeurs de tube peuvent causer des lésions tissulaires lors de l'insertion ou lors de l'administration d'oxygène, en particulier quand ils sont placés trop profondément dans l'arbre bronchique [61]. La technique originale consistant à rechercher activement le contact avec les anneaux trachéaux lors de l'insertion de guides longs est ainsi formellement déconseillée, au vu de son haut potentiel lésionnel.

L'utilisation de dispositifs réutilisables parait plus sûre d'un prime abord, néanmoins de rares cas de contamination par des microorganismes pathogéniques ont été décrits [62-64]. Le souci d'une efficacité moindre des instruments à usage unique reste présent, pendant que le risque cliniquement significatif de transmissions d'infections par leurs biais n'a pas été prouvé à ce jour.

\section{L'aide idéale à l'intubation}

L'objet idéal, nécessaire à toute intubation, n'existe pas. Il devrait être aisément disponible, à usage unique, simple à stocker et à transporter, facile et intuitif à utiliser ainsi que bon marché. De plus il devrait être suffisamment rigide pour maintenir sa forme après avoir été préformé (effet mémoire), mais suffisamment souple afin de ne pas causer de lésions dans les voies aériennes. Son extrémité distale devrait être souple et angulée afin de faciliter un positionnement simple - même à l'aveugle - sous l'épiglotte. II devrait être capable de donner un feedback du bon positionnement. Finalement, il devrait permettre une oxygénation rapide en cas d'urgence ou continue en cas d'essais prolongés d'intubation. Le mandrin idéal devrait également être compatible avec l'utilisation concomitante d'une multitude de techniques différentes (laryngoscopie directe ou indirecte type vidéolaryngoscopie) et il devrait permettre la mobilisation de l'extrémité distale sous visualisation directe. Différents prototypes de nouveaux mandrins sont actuellement en voie d'évaluation et devront prouver leur utilité dans des études cliniques. Des premiers résultats semblent prometteurs mais restent à être validés dans d'autres centres [65].

\section{Conclusion}

Lorsque la visualisation de l'orifice trachéal s'avère difficile, tous les efforts doivent être entrepris afin de guider un tube endotrachéal entre les cordes vocales, tout en minimisant le temps et le potentiel lésionnel sur les voies aériennes. L'utilisation précoce de longs guides et de mandrins, associés à une connaissance de leurs caractéristiques, indications, techniques d'utilisation et de leurs complications spécifiques, est primordiale afin de maximiser le taux de succès dans la prise en charge des voies aériennes.

\section{Déclaration de liens d'intérêts}

Sina Grape déclare ne pas avoir de liens d'intérêts.

Patrick Schoettker a participé au développement du SGuide Guide Long pour intubation, pour la firme VBM Medical (www.vbm-medical.com).

\section{Références}

[1] Hove LD, Steinmetz J, Christoffersen JK, Moller A, Nielsen J, Schmidt H. [Closed claims analysis of deaths related to anesthesia in the period 1996-2004 registered by the Danish Patient Insurance Association]. Ugeskrift for laeger 2007;169(34):2780-1. 
[2] Peterson GN, Domino KB, Caplan RA, Posner KL, Lee LA, Cheney FW. Management of the difficult airway: a closed claims analysis. Anesthesiology 2005;103(1):33-9.

[3] Cook TM, Woodall N, Frerk C, Fourth National Audit P. Major complications of airway management in the UK: results of the Fourth National Audit Project of the Royal College of Anaesthetists and the Difficult Airway Society. Part 1: anaesthesia. Br J Anaesth 2011;106(5):617-31, http://dx.doi.org/10. 1093/bja/aer058.

[4] Stasiuk RB. Improving styletted oral tracheal intubation: rational use of the OTSU. Can J Anaesth 2001;48(9):911-8, http://dx.doi.org/10.1007/BF03017359.

[5] Kidd JF, Dyson A, Latto IP. Successful difficult intubation. Use of the gum elastic bougie. Anaesthesia 1988;43(6): 437-8.

[6] Marshall SD, Pandit JJ. Radical evolution: the 2015 Difficult Airway Society guidelines for managing unanticipated difficult or failed tracheal intubation. Anaesthesia 2016;71(2):131-7, http://dx.doi.org/10.1111/anae.13354.

[7] Apfelbaum JL, Hagberg CA, Caplan RA, Blitt CD, Connis RT, Nickinovich DG, et al. Practice guidelines for management of the difficult airway: an updated report by the American Society of Anesthesiologists Task Force on Management of the Difficult Airway. Anesthesiology 2013;118(2):251-70, http://dx.doi.org/10.1097/ALN.0b013e31827773b2.

[8] Langeron 0, Bourgain JL, Laccoureye O, Legras A, Orliaguet G. [Difficult airway algorithms and management: question 5 . Societe Francaise d'Anesthesie et de Reanimation]. Ann Fr Anesth Reanim 2008;27(1):41-5, http://dx.doi.org/10.1016/ j.annfar.2007.10.025.

[9] Hartmannsgruber MW, Rosenbaum SH. Safer endotracheal tube exchange technique. Anesthesiology 1998;88(6):1683.

[10] Mort TC. Tracheal tube exchange: feasibility of continuous glottic viewing with advanced laryngoscopy assistance. Anesth Analg 2009;108(4):1228-31, http://dx.doi.org/10.1213/ ane.0b013e3181990a82.

[11] Lambotte P, Menu H, Guermouche T, Boufflers E, Ferri J, Krivosic-Horber R. [Intraoperative exchange of the endotracheal tube using the Cook C-CAE airway exchange catheter]. Ann Fr Anesth Reanim 1998;17(10):1235-8.

[12] Biro P, Priebe HJ. Staged extubation strategy: is an airway exchange catheter the answer? Anesth Analg 2007;105(5): 1182-5, http://dx.doi.org/10.1213/01.ane.0000286234. 96551.67.

[13] Batuwitage B, McDonald A, Nishikawa K, Lythgoe D, Mercer S, Charters P. Comparison between bougies and stylets for simulated tracheal intubation with the CMAC D-blade videolaryngoscope. Eur J Anaesthesiol 2014, http://dx.doi.org/10.1097/EJA.0000000000000070.

[14] Nielsen AA, Hope CB, Bair AE. GlideScope videolaryngoscopy in the simulated difficult airway: bougie vs standard stylet. West J Emerg Med 2010;11(5):426-31.

[15] Wadman M, Nicholas TA, Bernhagen MA, Kuper GM, Miljkovic $\mathrm{N}$, Schmidt S, et al. Use of a malleable bougie and curved forceps with videolaryngoscopy in airway management training in a cadaver model - a pilot study. Stud Health Technol Info 2012;173:537-9.

[16] Henderson JJ. Development of the "gum-elastic bougie". Anaesthesia 2003;58(1):103-4.

[17] Nolan JP, Wilson ME. An evaluation of the gum elastic bougie. Intubation times and incidence of sore throat. Anaesthesia 1992;47(10):878-81.

[18] Maclean J, Tripathy D, Parthasarathy S, Ravishankar M. Comparative evaluation of gum-elastic bougie and introducer tool as aids in positioning of ProSeal laryngeal mask airway in patients with simulated restricted neck mobility. Indian J Anaesth 2013;57(3):248-52, http://dx.doi.org/10.4103/ 0019-5049.115604.
[19] Brimacombe J, Keller C. Gum elastic bougie-guided insertion of the LMA-ProSeal in occult tonsillar hypertrophy. Can J Anaesth 2004;51(8):859, http://dx.doi.org/10.1007/BF03018475.

[20] Paul A, Gibson AA, Robinson OD, Koch J. The traffic light bougie: a study of a novel safety modification. Anaesthesia 2014;69(3):214-8, http://dx.doi.org/10.1111/anae.12522.

[21] Campbell J. Traffic light bougie: what is the safe zone? Anaesthesia 2014;69(5):514-5, http://dx.doi.org/10.1111/ anae. 12699.

[22] Evans H, Hodzovic I, Latto IP. Tracheal tube introducers: choose and use with care. Anaesthesia 2010;65(8):859, http://dx. doi.org/10.1111/j.1365-2044.2010.06432.x.

[23] Sorbello M, Frova G. Frova introducer: neither a stylet nor simply an introducer. Anaesthesia 2008;63(9):1010-1, http://dx.doi.org/10.1111/j.1365-2044.2008.05661_1.x [author reply 1011-1013].

[24] Muallem M. Endotracheal tube introducer: an aid for the difficult airway. Middle East J Anaesthesiol 2000;15(6):687-92.

[25] Muallem MK, Azar MS, Gerges FJ, Nasr VG, Baraka A. Muallem endo-tracheal tube introducer: (METTI)-an aid for the difficult airway. Middle East J Anaesthesiol 2005;18(2):385-9.

[26] Mort TC, Braffett BH. Conventional versus video laryngoscopy for tracheal tube exchange: glottic visualization, success rates, complications, and rescue alternatives in the highrisk difficult airway patient. Anesth Analg 2015;121(2):440-8, http://dx.doi.org/10.1213/ANE.0000000000000825.

[27] McLean S, Lanam CR, Benedict W, Kirkpatrick N, Kheterpal S, Ramachandran SK. Airway exchange failure and complications with the use of the Cook Airway Exchange Catheter(R): a single center cohort study of 1177 patients. Anesth Analg 2013;117(6):1325-7, http://dx.doi.org/10.1213/ANE. 0b013e3182a7cd3d.

[28] Choi EK, Kim JE, Soh SR, Kim CK, Park WK. Usefulness of a $\operatorname{Cook}(R)$ airway exchange catheter in laryngeal mask airway-guided fiberoptic intubation in a neonate with Pierre Robin syndrome - a case report. Korean J Anesthesiol 2013;64(2):168-71, http://dx.doi.org/10.4097/kjae.2013. 64.2.168.

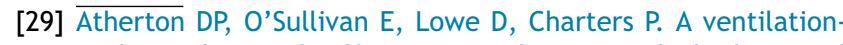
exchange bougie for fibreoptic intubations with the laryngeal mask airway. Anaesthesia 1996;51(12):1123-6.

[30] Heard AM, Lacquiere DA, Riley RH. Manikin study of fibreoptic-guided intubation through the classic laryngeal mask airway with the Aintree intubating catheter vs the intubating laryngeal mask airway in the simulated difficult airway. Anaesthesia 2010;65(8):841-7, http://dx.doi.org/10.1111/j.1365-2044.2010.06412.x.

[31] Malcharek MJ, Rockmann K, Zumpe R, Sorge O, Winter V, Sablotzki A, et al. Comparison of Aintree and Fastrach techniques for low-skill fibreoptic intubation in patients at risk of secondary cervical injury: a randomised controlled trial. Eur J Anaesthesiol 2014;31(3):153-8, http://dx.doi.org/10.1097/ EJA.0b013e328365ae49.

[32] Cook TM, Seller C, Gupta K, Thornton M, O'Sullivan E. Nonconventional uses of the Aintree Intubating Catheter in management of the difficult airway. Anaesthesia 2007;62(2):169-74, http://dx.doi.org/10.1111/j.1365-2044.2006.04909.x.

[33] Difficult Airway Society Extubation Guidelines G, Popat M, Mitchell V, Dravid R, Patel A, Swampillai C, et al. Difficult Airway Society Guidelines for the management of tracheal extubation. Anaesthesia 2012;67(3):318-40, http: //dx.doi.org/10.1111/j.1365-2044.2012.07075.x.

[34] Singh M, Kapoor D, Singh J. Extubation aid in double lumen tube exchange in difficult airway cases operated for thoracic surgeries. J Anaesthesiol Clin Pharmacol 2013;29(1):125, http://dx.doi.org/10.4103/0970-9185.105823.

[35] Francon D, Jaber S, Pean D, Bally B, Marciniak B. [Difficult extubation: extubation criteria and management of 
risk situations: question 6 . Societe française d'anesthesie et de reanimation]. Ann Fr Anesth Reanim 2008;27(1):46-53, http://dx.doi.org/10.1016/j.annfar.2007.10.026.

[36] Loudermilk EP, Hartmannsgruber M, Stoltzfus DP, Langevin PB. A prospective study of the safety of tracheal extubation using a pediatric airway exchange catheter for patients with a known difficult airway. Chest 1997;111(6):1660-5.

[37] Corso RM, Cattano D, Maitan S. Experience using a new staged extubation kit in patients with a known difficult airway. Anaesth Intensive Care 2015;43(1):137-8.

[38] Levitan RM, Pisaturo JT, Kinkle WC, Butler K, Everett WW. Stylet bend angles and tracheal tube passage using a straight-to-cuff shape. Acad Emerg Med 2006;13(12):1255-8, http://dx.doi.org/10.1197/j.aem.2006.06.058.

[39] Schoettker P. The orotracheal tube dance. Eur J Anaesthesiol 2015;32(6):443-4, http://dx.doi.org/10.1097/EJA. 0000000000000134.

[40] Rohsbach C, Wirth S, Lenz K, Priebe H. Survey on the current management of rapid sequence induction in Germany. Minerva anestesiol 2013;79(7):716-26.

[41] Ridgway S, Hodzovic I, Woollard M, Latto IP. Prehospital airway management in ambulance services in the United Kingdom. Anaesthesia 2004;59(11):1091-4, http://dx.doi.org/10.1111/ j.1365-2044.2004.03965.x.

[42] Jones PM, Loh FL, Youssef HN, Turkstra TP. A randomized comparison of the GlideRite $((R))$ Rigid Stylet to a malleable stylet for orotracheal intubation by novices using the GlideScope((R)). Can J Anaesth 2011;58(3):256-61, http: //dx.doi.org/10.1007/s12630-010-9440-z.

[43] Mahajan R, Shafi F, Sharma A. Use of Schroeder directional stylet to enhance navigability during nasotracheal intubation. J Anesth 2010;24(1):150-1, http://dx.doi.org/10. 1007/s00540-009-0838-0.

[44] Xue FS, Liao X, Liu JH, Yuan YJ, Wang Q. Performance of the GlideRite((R)) Rigid Stylet and malleable stylet for tracheal intubation by novices using the GlideScope $((R))$ videolaryngoscope. Can J Anaesth 2011;58(7):660, http://dx.doi.org/10.1007/s12630-011-9503-9 [author reply 661].

[45] Lomax SL, Johnston KD, Marfin AG, Yentis SM, Kathawaroo $S$, Popat MT. Nasotracheal fibreoptic intubation: a randomised controlled trial comparing the GlideRite(R) (Parker-Flex(R) Tip) nasal tracheal tube with a standard pre-rotated nasal RAE tracheal tube. Anaesthesia 2011;66(3):180-4, http://dx.doi.org/10.1111/j.1365-2044.2011.06621.x.

[46] Detave M, Shiniara M, Leborgne JM. [Use of Eschmann's gum elastic bougie in difficult orotracheal intubation, an audit over eight years of clinical practice]. Ann $\mathrm{Fr}$ Anesth Reanim 2008;27(2):154-7, http://dx.doi.org/10.1016/ j.annfar.2007.11.006.

[47] Jabre P, Combes X, Leroux B, Aaron E, Auger H, Margenet A, et al. Use of gum elastic bougie for prehospital difficult intubation. Am J Emerg Med 2005;23(4):552-5.

[48] Marson BA, Anderson E, Wilkes AR, Hodzovic I. Bougie-related airway trauma: dangers of the hold-up sign. Anaesthesia 2014;69(3):219-23, http://dx.doi.org/10.1111/anae.12534.

[49] Hodzovic I, Latto IP, Wilkes AR, Hall JE, Mapleson WW. Evaluation of Frova, single-use intubation introducer, in a manikin.
Comparison with Eschmann multiple-use introducer and Portex single-use introducer. Anaesthesia 2004;59(8):811-6, http://dx.doi.org/10.1111/j.1365-2044.2004.03809.x.

[50] Kadry M, Popat M. Pharyngeal wall perforation-an unusual complication of blind intubation with a gum elastic bougie. Anaesthesia 1999;54(4):404-5.

[51] Arndt GA, Cambray AJ, Tomasson J. Intubation bougie dissection of tracheal mucosa and intratracheal airway obstruction. Anesth Analg 2008;107(2):603-4, http://dx.doi.org/10.1213/ ane.0b013e318176fe36.

[52] Sahin M, Anglade D, Buchberger M, Jankowski A, Albaladejo P, Ferretti GR. Case reports: iatrogenic bronchial rupture following the use of endotracheal tube introducers. Can J Anaesth 2012;59(10):963-7, http://dx.doi.org/10. 1007/s12630-012-9763-z.

[53] Staikou C, Mani AA, Fassoulaki AG. Airway injury caused by a Portex single-use bougie. J Clin Anesth 2009;21(8):616-7, http://dx.doi.org/10.1016/j.jclinane.2009.03.006.

[54] Smith BL. Haemopneumothorax following bougie-assisted tracheal intubation. Anaesthesia 1994;49(1):91.

[55] Cooper RM. Complications associated with the use of the GlideScope videolaryngoscope. Can J Anaesth 2007;54(1):54-7 [doi: 54/1/54 (pii)].

[56] Magboul MM, Joel S. The video laryngoscopes blind spots and possible lingual nerve injury by the Gliderite rigid stylet-case presentation and review of literature. Middle East J Anesthesiol 2010;20(6):857-60.

[57] Choo MK, Yeo VS, See JJ. Another complication associated with videolaryngoscopy. Can J Anaesth 2007;54(4):322-4, http://dx.doi.org/10.1007/BF03022782.

[58] Chalhoub V, Richa F, El-Rassi I, Dagher C, Yazbeck P. Pulmonary migration of a fragment of plastic coating sheared from a stylet. J emerg med 2013;44(6):1097-100, http://dx.doi.org/10. 1016/j.jemermed.2012.11.004.

[59] Schaffranietz L, Graz F, Tamke A. [Intratracheal foreign body caused by a sheared endotracheal intubation stylet]. Der Anaesthesist 2009;58(12):1223-5, http://dx.doi.org/10.1007/ s00101-009-1641-x.

[60] Gardner M, Janokwski S. Detachment of the tip of a gum-elastic bougie. Anaesthesia 2002;57(1):88-9.

[61] Axe R, Middleditch A, Kelly FE, Batchelor TJ, Cook TM. Macroscopic barotrauma caused by stiff and soft-tipped airway exchange catheters: an in vitro case series. Anesth Analg 2015;120(2):355-61, http://dx.doi.org/10.1213/ANE. 0000000000000569 .

[62] Miller DM, Youkhana I, Karunaratne WU, Pearce A. Presence of protein deposits on "cleaned" re-usable anaesthetic equipment. Anaesthesia 2001;56(11):1069-72.

[63] Cupitt JM. Microbial contamination of gum elastic bougies. Anaesthesia 2000;55(5):466-8.

[64] Rowley E, Dingwall R. The use of single-use devices in anaesthesia: balancing the risks to patient safety. Anaesthesia 2007;62(6):569-74, http://dx.doi.org/10.1111/ j.1365-2044.2007.04995.x.

[65] Nkoulou CBI, Fournier N, Schoettker P. S-Guide versus Gliderite for videolaryngoscopic intubation of patients with simulated difficult airways. Eur J Anaesthesiol 2016;33(S54):430. 